\title{
HIGH POWER TEST OF A 30-GHZ PLANAR ACCELERATOR*
}

\author{
D. Yu, DULY Research Inc., Rancho Palos Verdes, CA 90275 \\ H. Henke, Technical University of Berlin, Germany \\ H. H. Braun, S. Döbert, W. Wuensch, CERN, Geneva, Switzerland
}

\begin{abstract}
A $30-\mathrm{GHz}$ muffin-tin, traveling-wave accelerating structure consisting of 37 cells was tested at high power using the CTF II at CERN. The structure was fabricated with conventional milling and brazing, including tuning holes at cavity roofs. No special surface preparation or treatment was done to the structure. A maximum peak power in excess of $100 \mathrm{MW}$ at a pulse width of 4 ns was transported through the structure before electron bursts were initiated. A maximum accelerating gradient of 60 $\mathrm{MV} / \mathrm{m}$ was achieved with a peak RF power of $40 \mathrm{MW}$ at a pulse width of $8 \mathrm{~ns}$.
\end{abstract}

\section{INTRODUCTION}

For high energy linear colliders, planar accelerating structures may have significant advantages [1] over conventional cylindrical structures. Some of these are listed below:

Fabrication advantage - Modern microfabrication techniques such as LIGA can be used to produce highfrequency, high-tolerance planar structures with large aspect ratios. The new fabrication techniques may reduce the cost of mass producing planar accelerating structures, making them attractive also for future, ultra compact commercial accelerators.

Wakefield advantage - Planar structures have smaller transverse wakefields than traditional cylindrical structures, rendering them suitable for long accelerators employing a multi-bunch acceleration scheme. By opening the cavity side walls it is possible to propagate one of the transverse wakefields out of the structure.

Beamsstrahlung advantage - Flat beams with a large aspect ratio are useful to reduce Beamsstrahlung in the final focus region of a linear collider. Planar structures offer the possibilities of providing a flat field in the transverse dimensions and accelerating a flat beam [2].

As part of a DOE SBIR project to investigate the feasibility of using planar accelerating structures for linear colliders and compact accelerators, we constructed a completely engineered, 30-GHz, 37-cell, muffin-tin structure, and high power tested it at CERN. The successful experiment offers a proof of principle to accelerate a round beam in a simple planar structure. While the electromagnetic properties of the structure, such as elastance and shunt impedance, wakefields, and eigenmodes are directly scaleable to any frequency, there are non-scaling quantities such as breakdown, dark current and ohmic heating. Our experiments also included

\footnotetext{
* Work supported by DOE SBIR Grant No. DE-FG03-96ER82213.
}

measurements of dark currents and breakdown thresholds of the $30-\mathrm{GHz}$ structure.

\section{DESIGN AND FABRICATION}

The frequency choice for our first-time demonstration of a high-gradient, planar accelerator is largely dictated by the highest-frequency, highest-power RF source currently available in the world. The $29.985-\mathrm{GHz} \mathrm{RF}$ source at the CLIC Test Facility (CTF II) [3] at CERN, Switzerland is uniquely suited for this proof-of-principle experiment. The design and fabrication techniques of our 37-cell, traveling-wave, constant-impedance, muffin-tin structure are similar to those which were reported in an earlier PAC paper [4], except resonant frequencies of the individual cells in the new structure are tuneable. An earlier structure, which we also tested at CERN, was detuned by about $250 \mathrm{MHz}$ after brazing and consequently was able to only meagerly accelerate an electron beam [5]. By contrast, the frequency of the new tuneable structure came within $25 \mathrm{MHz}$ of the operating frequency of the RF source, and achieved more than $95 \%$ of the predicted acceleration.

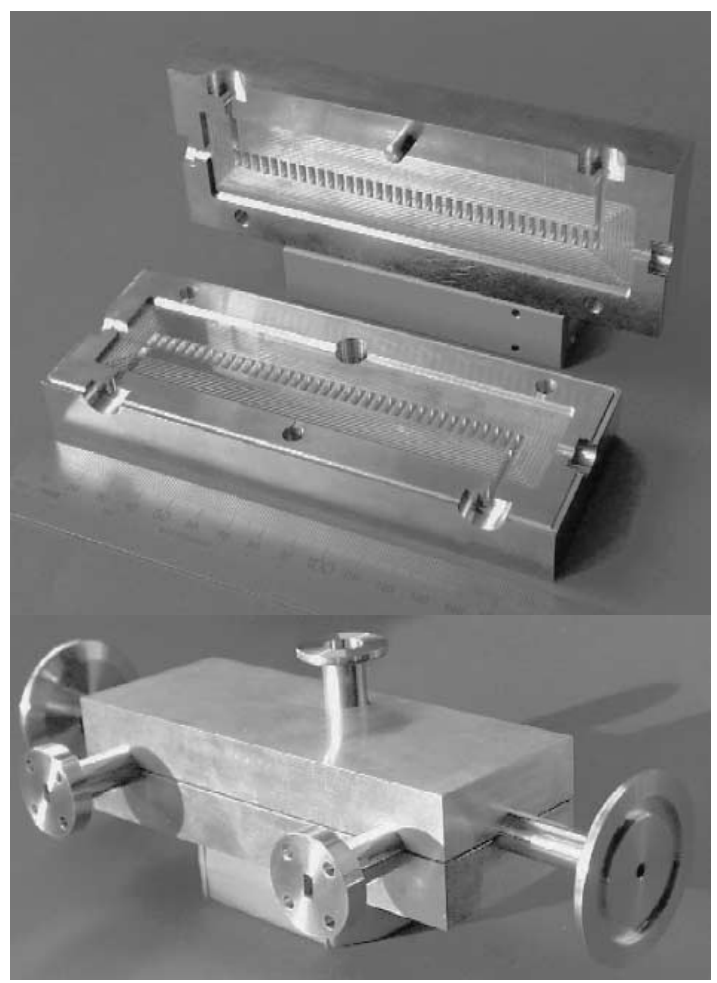

Figure 1: Interior and exterior of a 37-cell planar accelerator (active length $=12.3 \mathrm{~cm}$ ). 
The overall geometry of the structure including the RF couplers are shown in Fig 1. The main structure consists of two identical pieces which are separately machined with conventional CNC milling, with no special surface finish. Alignment of the two halves of the structure is provided by 3 dowel pins. Frequency tuning is done by drilling a hole centered on the roof of each cell. Two brazing cycles are required for this structure: The first brazing bonds the two halves of the structures together, and the second brazing bonds the external ports ( $2 \mathrm{RF}$ ports, 1 vacuum port and 2 beam ports) to the structure.

\section{FREQUENCY TUNING}

Fig 2 shows a GdfidL simulation including the tuning holes for the structure. Frequency shift is determined primarily by the radius of the hole, which in all cases is smaller than cutoff. Since the field is evanescent inside the hole, the depth is not important. The holes are drilled from inside and do not break the vacuum boundary. Fig 3 is a plot of the hole size versus frequency for the structure from this simulation.

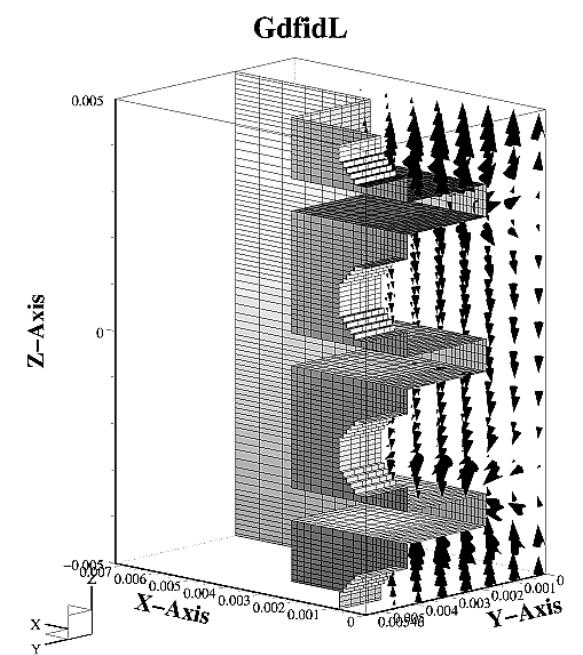

Figure 2: GdfidL model of tuned muffin-tin structure.

Frequency vs Hole Radius for a Muffin Tin

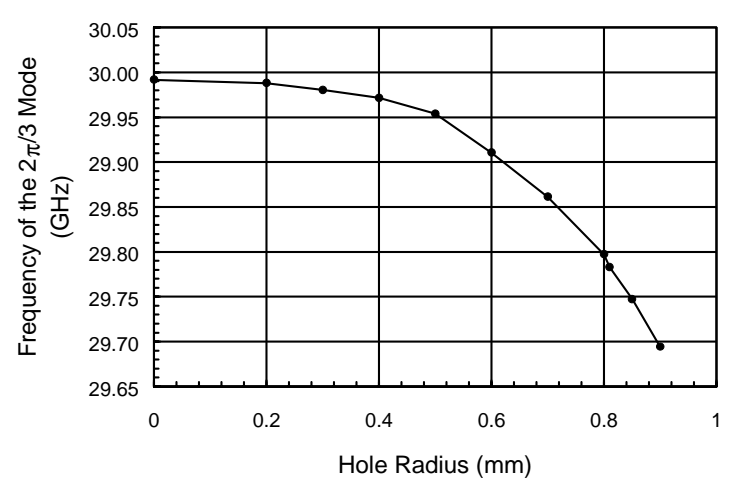

Figure 3: Tuning curve for muffin-tin structure.

\section{HIGH POWER TESTING}

A 30-GHz, high-power test facility, CTF II, has been developed by CERN [3] to test CLIC prototype accelerating structures. RF power is produced by decelerating a high-charge drive beam in a specially designed power extraction structure. This power was fed into the planar cavity which was installed in a second beam line providing a single, low-charge, electron bunch to probe the achieved accelerating gradients. The energy gain of the probe beam in the planar structure is measured with a magnetic spectrometer at the end of the beam line.

The incident, transmitted and reflected RF powers are measured via directional couplers. The $30-\mathrm{GHz}$ signals are down converted to $500 \mathrm{MHz}$ and digitized with a fast oscilloscope. The high-power test stand is equipped with a pair of wall current monitors on each side of the test structure to measure the intensity of spontaneous electron burst emitted during a breakdown event and to measure dark currents. Each pair of monitors is separated by about one meter; therefore the energy of these emitted electrons can be determined by measuring their time of flight. Vacuum gauges at different locations near the planar structure completed the diagnostics for this experiment.

The planar structure was installed and in-situ baked at $120{ }^{\circ} \mathrm{C}$ for two days, resulting in a pressure of the order of $10^{-8}$ mbar. The synchronous frequency was determined to be $30.009 \mathrm{GHz}$ by measuring the radiation spectrum excited by passing a short probe bunch through the structure. CTF II can provide RF pulses with widths of 4,8 or $16 \mathrm{~ns}$ at a repetition rate of $5 \mathrm{~Hz}$. Since the planar structure had a group velocity of $\mathrm{v}_{\mathrm{g}} / \mathrm{c}=11.2 \%$ corresponding to a filling time of almost $4 \mathrm{~ns}$, a pulse length of $8 \mathrm{~ns}$ was chosen for conditioning and probe beam acceleration. A typical RF pulse is shown in Fig 4.

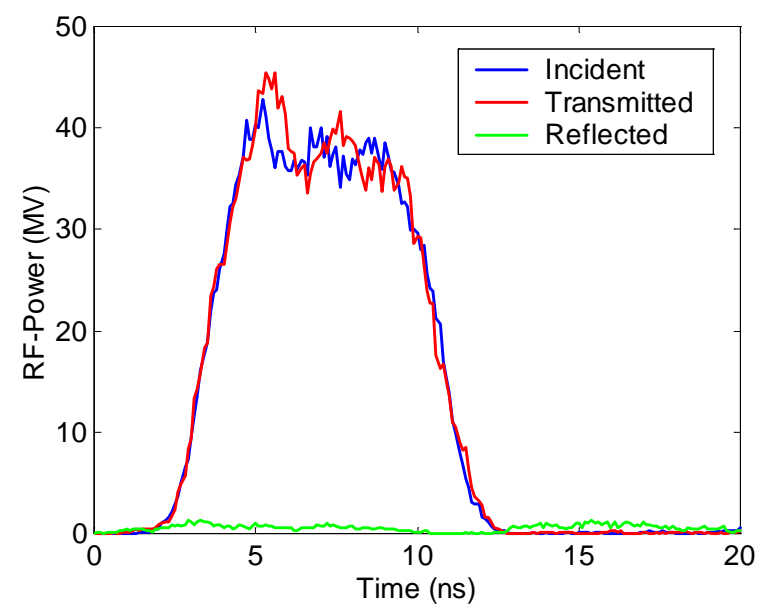

Figure 4: Typical measured incident, transmitted and reflected RF pulse used to condition the planar structure.

RF conditioning was done by watching the vacuum and wall current monitor signals while carefully raising the RF input power to avoid breakdown. An electrical breakdown event manifests itself by a certain amount of missing energy after accounting for the input and output powers, by a pressure rise, and by the emission of electron bursts emitted through the cavity beam aperture. The emission of electron bursts was found to be the most sensitive indicator for electrical breakdown events, while significant pressure rises are usually observed only at the beginning of conditioning steps. In a typical breakdown event, the pulse of the transmitted power was 
considerably shortened, but there was no major increase in the reflected power. Breakdown events are accompanied by massive electron emission observed upstream of the cavity, with peak currents often exceeding $100 \mathrm{~mA}$. The amount of electron emission is correlated with the missing energy in the corresponding RF pulse as shown in Fig 5. The average energy of these electrons was measured to be around $300 \mathrm{keV}$ at an average accelerating gradient of $60 \mathrm{MV} / \mathrm{m}$. Dark currents, i.e. stable electron emission every pulse even with no breakdown events, were absent below $90 \mathrm{MV} / \mathrm{m}$.

One of the important objectives of the high power test of the planar structure was to demonstrate its capability for synchronous electron acceleration. The expected relation between input power and energy gain through the structure, from GdfidL simulations, is $\Delta \mathrm{E}(\mathrm{MV})=$ $1.2 \sqrt{ } \mathrm{P}_{0}(\mathrm{MW})$. We demonstrated electron acceleration with a $30 \mathrm{GHz}$ planar structure up to an average gradient of $60 \mathrm{MV} / \mathrm{m}$ using an $8 \mathrm{~ns} \mathrm{RF}$ pulse. The measured energy gain of the probe beam was in excellent agreement with the expectations from the power measurements as shown in Fig 6.

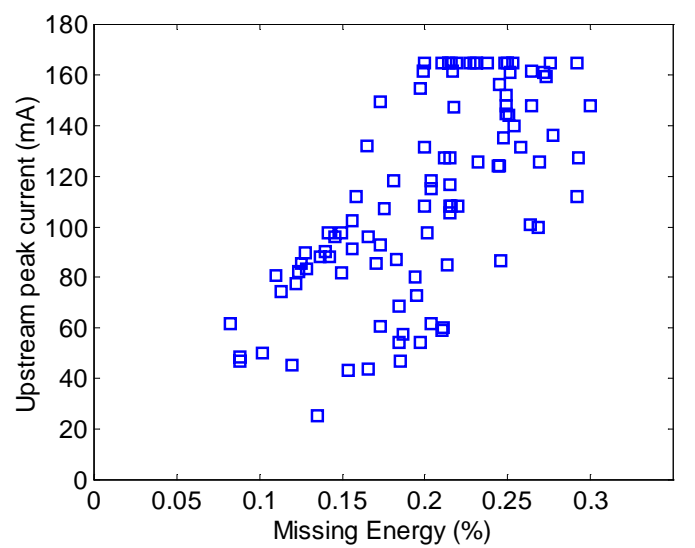

Figure 5: Correlation between missing energy and upstream current bursts emitted during breakdown.

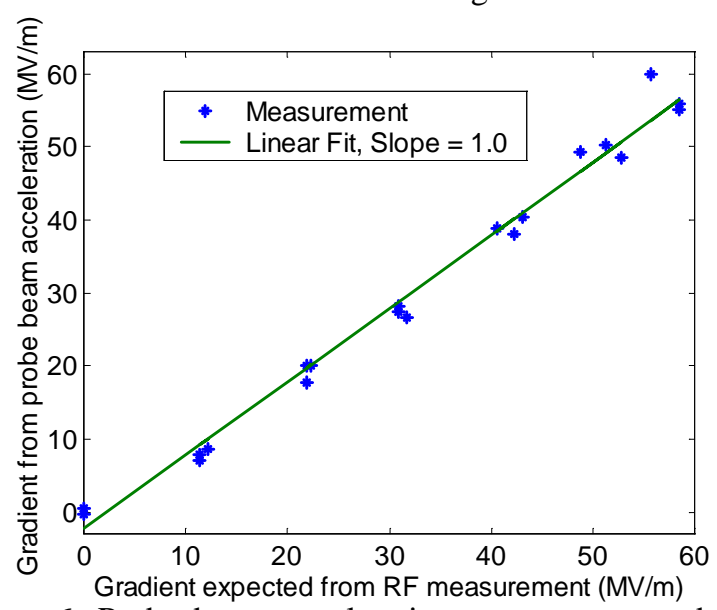

Figure 6: Probe beam acceleration versus measured RF power.

The achievable gradient without breakdowns was investigated also as a function of the RF pulse length. At a pulse length of $16 \mathrm{~ns}$ breakdown occurred above $50 \mathrm{MV} / \mathrm{m}$ and at a pulse length of $4 \mathrm{~ns}$ above $95 \mathrm{MV} / \mathrm{m}$ corresponding to an input power of nearly $100 \mathrm{MW}$. A cubic root law describes this dependence best (see Fig 7.). The maximal gradients quoted above are not the final result of a long conditioning procedure because of a limited time for the experiment (one week) and the repetition rate of CTF II.

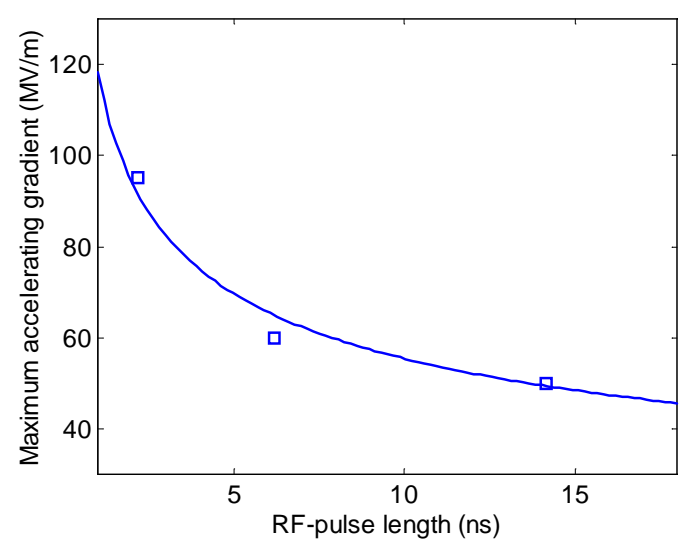

Figure 7: Dependence of the maximal achievable gradient without breakdown on RF pulse length. The continuous curve is a cubic root fit to the data.

The question of damage due to RF conditioning is currently an important subject especially for highfrequency and high-gradient accelerating structures. In order to monitor damage due to RF during the conditioning process, the synchronous frequency of the structure from the RF induced by a single bunch, and the consistency of the energy gain prediction with the actual, measured energy gain, was checked frequently. No sign of damage was found from these measurements, precise S-parameter measurements done with a network analyser before and after the high power test also indicated no damage had occurred. Nevertheless we also did an inspection with an optical endoscope. Looking into the structure gave the impression that the irises of the first three cells suffered slight damage in the high surface gradient region in the middle of the iris. A clearer statement is currently not possible because of the limited resolution and imaging quality of the endoscope. The ratio of surface gradient to accelerating gradient was estimated to $\mathrm{E}_{\text {sur }} / \mathrm{E}_{\mathrm{acc}} \approx 6$ in the middle of the irises. Therefore the surface gradients exceeded $300 \mathrm{MV} / \mathrm{m}$, which is similar to the surface gradients that caused damage in CLIC prototype structures [6].

\section{REFERENCES}

[1] D. $\mathrm{Yu}$ et al., Proc. $6^{\text {th }}$ Advanced Accelerator Workshop, Lake Geneva, Wisconsin, 1994, p. 800.

[2] D. Yu et al., these proceedings, PAC2001.

[3] H. H. Braun et al., Proc. $7^{\text {th }}$ EPAC2000, Vienna, Austria 2000, 48.

[4] R. Merte, H. Henke, M. Peikert, D. Yu, Proc. PAC, New York, 1999, p. 815.

[5] R. Merte et al., Proc. $7^{\text {th }}$ EPAC, Vienna, 2000.

[6] H. H. Braun, S. Döbert, L. Groening, I. Wilson, F. Zhou, CLIC Note 475, 2001. 